\title{
La filière oléagineuse se mobilise autour de la problématique des résidus d'insecticides
}

\author{
Sylvie DAUGUET ${ }^{1}$ \\ Florence LACOSTE ${ }^{2}$ \\ Bernard TICOT ${ }^{3}$ \\ Jean-Philippe LOISON ${ }^{1}$ \\ Jacques EVRARD ${ }^{1}$ \\ Bouchaïb BOUCHTANE ${ }^{4}$ \\ Bénédicte SOULET ${ }^{2}$ \\ ${ }^{1}$ CETIOM, Service transformation \\ et valorisation des graines oléagineuses \\ 11 rue Monge Parc Industriel 33600 Pessac \\ 2 ITERG, Institut des Corps Gras, \\ 11 rue Monge Parc Industriel 33600 Pessac \\ ${ }^{3}$ ONIDOL, 12 avenue George V 75008 Paris \\ ${ }^{4}$ CETIOM, Laboratoire d'Ardon, \\ 270 avenue de la Pomme de Pin \\ BP 90635, 45166 Olivet Cedex
}

\section{Introduction}

Les crises récentes de la vache folle et de la dioxine et leur traitement par les médias ont nuit gravement à la confiance des consommateurs vis-à-vis du secteur agricole et agroalimentaire. Restaurer cette confiance en garantissant la sécurité des aliments doit être une préoccupation pour chaque maillon des filières agricoles. Lorsqu'une crise alimentaire survient en effet, c'est l'ensemble de la filière agricole concernée, de la production à la distribution, qui est pénalisée. En parallèle, la Commission européenne a publié plusieurs textes législatifs regroupés sous le terme "Paquet Hygiène » qui précisent que les producteurs agricoles, fabricants d'aliments du bétail et industries agro-alimentaires sont responsables de la sécurité sanitaire de leur production. La traçabilité est devenue obligatoire ainsi que la mise en œuvre d'une analyse de risques par tous les opérateurs de la chaîne alimentaire. Cette priorité donnée à la sécurité sanitaire se traduit au niveau de la filière oléagineuse par la mise en place d'actions concertées visant à évaluer le niveau de contamination de la production à la distribution, à améliorer les méthodes d'analyse et à améliorer les pratiques. Ainsi l'ensemble de la filière oléagineuse se mobilise autour de la sécurité sanitaire.

\begin{abstract}
Oilseed chain professionals are coordinated through a food safety survey plan, in order to have a realistic picture of contaminations in oilseed products. Pesticide residues are found in seeds and crude oils: they are mainly organophosphate insecticides (pirimiphos-methyl, dichlorvos, malathion) used for empty storage premises treatment and for stored cereal grains treatment. Even if studies showed that pests can be found in stored oilseeds, French regulation does not authorize these insecticides on stored oilseeds, which have however an affinity for liposoluble chemicals and can pick these up from storage environment. This uptake of insecticide residues by oilseeds from their storage environment can lead to levels that can exceed regulatory limits. Works are in progress in order to improve and standardize analytical methods for pesticide residues in oilseeds and oils.
\end{abstract}

Key words: oilseeds, vegetable oil, contaminants, pesticide residues, survey plan

\section{Une filière qui s'autocontrôle}

Afin d'avoir une meilleure connaissance sur la présence d'éventuels contaminants dans les produits issus d'oléagineux, les opérateurs de la filière ont décidé de mettre en place un Plan de surveillance de la qualité sanitaire des oléagineux dès 2003 (figure 1). Son objectif est de créer une base de données mutualisée sur les contaminants du tournesol, du colza et du soja. Cette base est alimentée par les données d'autocontrôle des industriels et des organismes stockeurs qui adhèrent à ce plan, ainsi que par les campagnes d'analyses sanitaires menées sur graines, tourteaux et huiles par le Cetiom, l'Onidol et l'Iterg de 2003 à 2006. Un comité de pilotage, composé des fédérations professionnelles de la filière et d'experts scientifiques de I'Inra, définit annuellement les contaminants à surveiller en priorité, contribue à l'interprétation des résultats et garantit le bon fonctionnement du plan de surveillance. Le

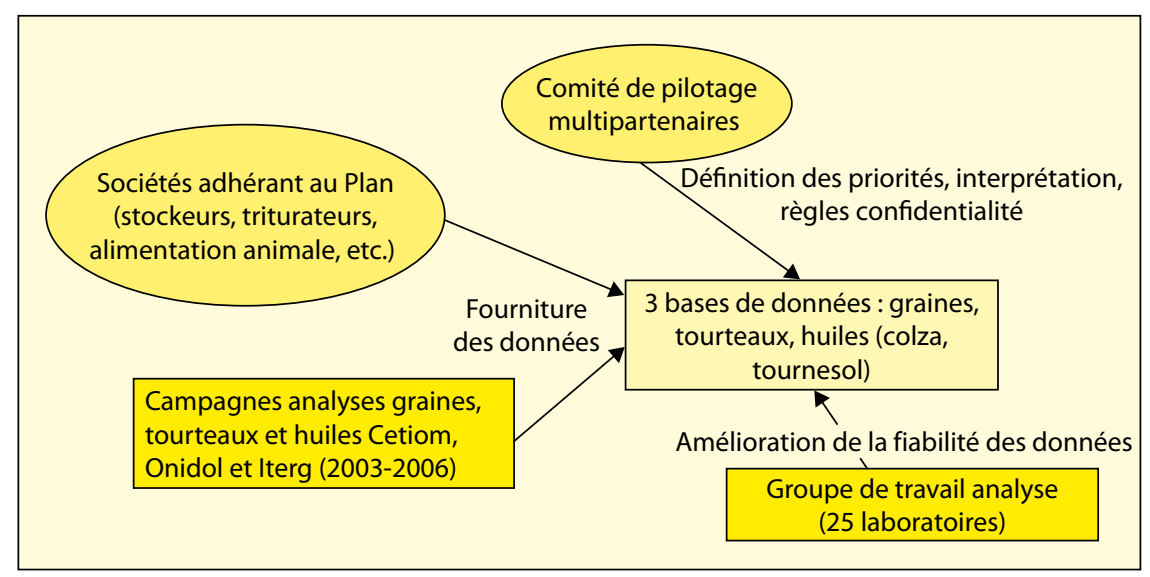

Figure 1. Organisation du Plan de surveillance de la qualité sanitaire des oléagineux. 
conventionnement avec les entreprises a débuté courant 2004 et, à ce jour, 20 sociétés (collecte/stockage, industries de I'huilerie, fabricants d'aliments du bétail) participent au plan de surveillance dont le fonctionnement est régi par des règles de confidentialité. Ainsi, chaque adhérent qui fournit des données reçoit annuellement un rapport individuel où il peut se positionner par rapport à l'ensemble des partenaires: pour un investissement modéré en analyses, il peut donc avoir accès à une base de données riche, lui permettant de faire une analyse de risque pour son activité plus fine. Des rapports sur la récolte 2004 et sur la récolte 2005 ont déjà été édités, avec un nombre de données fournies par les adhérents qui a augmenté significativement entre les deux années. Les opérateurs de la filière adhèrent globalement bien à cette démarche; cependant un effort devra être fait auprès des organismes de collecte-stockage afin de les inciter à participer en nombre.

Les campagnes d'analyses sanitaires menées depuis 2003 par le Cetiom, l'Iterg et l'Onidol ont permis d'avoir un premier état des lieux sur les contaminants présents dans les produits oléagineux. Les substances prioritairement recherchées ont été : résidus de produits phytopharmaceutiques (organophosphorés, organochlorés, pyréthrinoïdes), éléments traces métalliques (plomb, cadmium), mycotoxines (aflatoxine B1 essentiellement), salmonelles dans les tourteaux, HAP (hydrocarbures aromatiques polycycliques) dans les huiles. Ces enquêtes ont montré que les produits oléagineux sont en quasi-totalité conformes à la réglementation en vigueur. Cependant, on voit également que le raffinage est nécessaire pour éliminer les résidus des produits phytopharmaceutiques des huiles brutes.

\section{Les résidus d'insecticides}

Les molécules les plus fréquemment détectées dans les graines et huiles brutes sont: pyrimiphos-méthyl, dichlorvos, malathion, tous les trois des insecticides organophosphorés utilisés au stockage. Suite au process de trituration, ces molécules lipophiles se retrouvent essentiellement dans l'huile brute et très peu dans les tourteaux. Donc ce transfert dans I'huile s'accompagne d'une concentration d'un facteur de l'ordre de 2. Ces résidus d'insecticides sont retrouvés dans une majorité $d$ 'échantillons d'huiles brutes de colza et tournesol (70-80 \% des cas), et dans les graines (20 à $30 \%$ des cas), avec des teneurs relativement basses, en moyenne 0,1 à $0,25 \mathrm{mg} / \mathrm{kg}$ pour la somme des résidus de ces molécules dans les huiles brutes et des teneurs maximales pouvant aller jusqu'à $1 \mathrm{mg} / \mathrm{kg}$ (figure 2). Les LMR (limites maximales de résidus) dans les graines sont par-

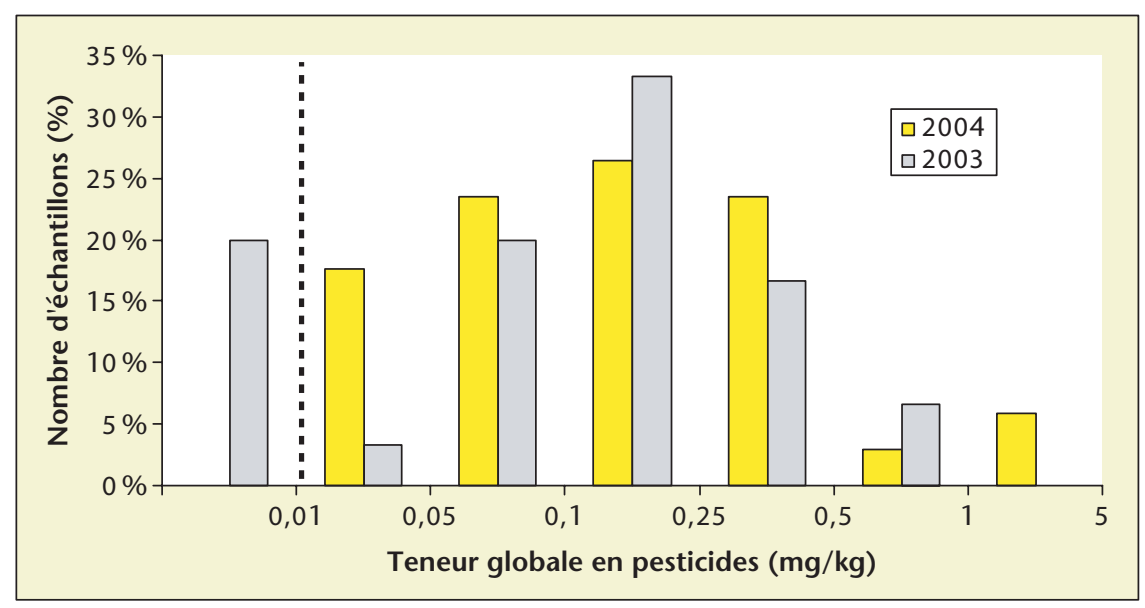

Figure 2. Résultats des enquêtes menées en 2003 et 2004 sur la présence de résidus de produits phytopharmaceutiques dans les huiles brutes de tournesol et colza (64 échantillons examinés) - Données Iterg.

fois dépassées, celles-ci étant très basses ou inexistantes (dans ce dernier cas, la limite de quantification ne doit pas être dépassée) du fait que ces insecticides ne sont pas autorisés en traitement direct des graines oléagineuses (tableau 1).

Dans les huiles raffinées, on ne retrouve plus ces résidus d'insecticides qui ont été éliminés (travaux de l'Iterg sur l'efficacité des étapes successives de raffinage sur l'élimination des molécules). II ne s'agit donc pas d'un problème de sécurité sanitaire du consommateur, mais plutôt d'une conformité par rapport à la réglementation.

L'origine de ces résidus d'insecticides du stockage (pyrimiphos-méthyl, malathion, dichlorvos) peut être des traitements directs des graines, accidentels ou volontaires, lorsque les teneurs retrouvées sont élevées (de l'ordre du $\mathrm{mg} / \mathrm{kg}$ ), même si ces cas sont de plus en plus rares grâce à la communication faite auprès des organismes stockeurs. Les teneurs retrouvées étant relativement basses, cela correspond plutôt à un « bruit de fond » du fait que les sites de stockage des oléagineux sont communs avec les céréales. Les graines oléagineuses sont donc en contact avec un environnement où ces insecticides sont présents de manière résiduelle. En effet, les insecticides utilisés au stockage se dégradent peu en milieu confiné contrairement aux produits appliqués en culture. Les installations de stockage, lorsqu'elles sont vides, ainsi que les matériels de transport du silo (convoyeurs, élévateurs, etc.), font l'objet de nettoyage et de traitements insecticides. Les graines oléagineuses par la suite peuvent être « contaminées » par contact avec les surfaces qui ont été traitées. D'autre part, des céréales qui sont traitées avec des insecticides peuvent contaminer les circuits. Une contamination croisée des grains via le matériel de manutention et de stockage peut faire apparaître alors des résidus posant des problèmes de commercialisation et de respect des contrats.

\section{Des insectes dans les graines oléagineuses stockées}

Contrairement à l'idée reçue que les graines oléagineuses ne sont pas sujettes aux infestations au stockage, des cas de présence d'insectes de stockage ont été signalés ces dernières années dans la filière oléagineuse par les opérateurs de stockage et de la trituration. N'ayant

Tableau 1. Limites maximales de résidus (LMR) des principales molécules insecticides utilisées au stockage sur graines oléagineuses et céréales $(\mathrm{mg} / \mathrm{kg})$.

\begin{tabular}{|lccc|}
\hline Substances actives & Tournesol et Soja & Colza & Céréales \\
\hline $\begin{array}{l}\text { malathion } \\
\text { dichlorvos }\end{array}$ & - & - & 8 \\
& 0,01 & 0,01 & 0,01 (Directive CE 09/11/2006, \\
pyrimiphos-méthyl & 0.05 & & 5 (10 sur son de blé) \\
chlorpyriphos-méthyl & 0.05 & - & 3 \\
deltaméthrine & 0.05 & 0.1 & 1 \\
bifenthrine & - & 0.01 & 0.5 \\
pyréthrines & - & - & 3 \\
phosphure d'hydrogène & - & - & $0.1(0.01$ sur farine) \\
\hline
\end{tabular}


aucun insecticide de contact autorisé en France pour la désinsectisation des oléagineux stockés, les organismes stockeurs (OS) peuvent avoir des difficultés à respecter la clause contractuelle d'absence d'insectes vivants dans les graines. Face à cette situation, le Cetiom a mené une étude sur trois ans pour dresser un état des lieux des problèmes d'insectes rencontrés et rechercher des voies de lutte: par la fumigation au gaz phosphine, par la mise en place de bonnes pratiques de stockage préventives.

Pour réaliser l'état des lieux des infestations, des échantillons de graines de tournesol et de colza ont été prélevés dans des silos de stockage (34 en 2002 et 121 en 2003) sur toute la zone de production française de ces cultures, en surface des tas au printemps, selon la méthode de dépistage orienté non aléatoire. Les échantillons ne sont donc pas représentatifs de la densité d'infestation moyenne des stocks de graines, établie par prélèvements réguliers en cours de vidange de cellules mais permettent de connaître les types d'insectes trouvés sur les oléagineux et leur fréquence $d$ 'infestation de la partie superficielle des masses de graines stockées. La fréquence d'infestation et la densité d'insectes ont été déterminées par tamisage, complétées par l'identification des espèces rencontrées.

Il est ressorti de ces travaux que les graines de colza sont relativement indemnes d'insectes, même si la présence d'acariens a été observée régulièrement, cette dernière ne posant pas de problème de commercialisation. En revanche, des insectes sont souvent présents dans les stocks de tournesol (près de $50 \%$ des cas), principalement des types ravageurs secondaires et mycophages qui ne s'attaquent pas directement aux graines et donc ne dégradent pas leur qualité. Cependant, ils gênent la com- mercialisation des lots et sont des indicateurs de mauvaises conditions de stockage.

Une enquête réalisée auprès des responsables de 85 organismes stockeurs a permis d'établir des relations entre les caractéristiques des sites de stockage, la conduite des opérations de stockage et le risque d'infestation par des insectes. Elle démontre que la mise en place de bonnes pratiques de conservation est primordiale pour éviter la présence d'insectes. Les principales conclusions sont les suivantes:

II y a corrélation entre absence d'insectes et basse teneur en eau.

Il y a corrélation entre présence de ravageurs secondaires (Tribolium castaneum, Oryzaephilus sp., Cryptolestes ferrugineus) et stockage à plat. La présence majoritaire d'insectes mycophages est corrélée positivement avec une température plutôt élevée.

Les fortes densités d'infestation sont surtout rencontrées dans des cellules métalliques plutôt qu'en béton.

À l'heure actuelle, seule la fumigation au gaz phosphine $\left(\mathrm{PH}_{3}\right)$ permet de lutter curativement contre les infestations $d$ 'insectes (encadré 1). Le nettoyage mécanique avant expédition offre une solution alternative intéressante et peu coûteuse. En effet, les insectes trouvés sur tournesol, secondaires et mycophages, vivent surtout en surface des graines. Ce procédé permet donc de les éliminer.

\section{Transfert de résidus d'insecticides du stockage par contact}

Comme nous l'avons expliqué précédemment, les graines oléagineuses sont contaminées par les résidus d'insecticides du stockage à de faibles teneurs car elles sont en contact avec un environnement où ces substances sont présentes de manière résiduelle. Afin d'évaluer l'importance de ces transferts par contact, le Cetiom a mené avec le soutien de l'Onidol une étude avec le Laboratoire national des denrées stockées (LNDS) de la protection des végétaux. Des plaques en métal de $200 \mathrm{~cm}^{2}$ ont été disposées aléatoirement sur des bâches en polyéthylène de $10 \mathrm{~m}^{2}$. Chaque bâche a été traitée avec du pyrimiphos-méthyl à la dose de 0,2 $\mathrm{g}$ de matière active par $\mathrm{m}^{2}$. Après 24 heures de séchage à l'air ambiant, les plaques traitées ont été récupérées et une monocouche de graines de colza et de grains de blé a été disposée dessus. La même manipulation a été reproduite le lendemain pour avoir un essai de répétition. Deux temps de contact entre les plaques et les graines ont été appliqués : 8 jours et 29 jours. Les résultats obtenus montrent qu'il y a bien un transfert de résidus de la plaque vers les graines et que cet échange semble se faire dans des proportions assez semblables pour les deux types de graines testées (tableau 2).

La différence entre les taux de transfert de pyrimiphos-méthyl du blé et du colza pourrait s'expliquer par les caractéristiques des graines (forme, structure de la cuticule).

L'extrapolation des résultats obtenus sur plaques à des cellules de stockage de type fermières montre que le risque de dépassement des $\operatorname{LMR}(0,050 \mathrm{mg} / \mathrm{kg}$ de pyrimiphos-méthyl) est bien réel (tableau 3).

D'après les calculs, plus une cellule est grande, plus le rapport volume de produit sur surface traitée est grand et ainsi plus on se met en sécurité par rapport au risque de dépassement de la LMR par transfert de résidus depuis les parois des cellules de stockage traitées. En cellule d'OS de grande capacité, le risque d'atteindre 50 ppb paraît donc minime, voire inexistant dans le respect des bonnes pratiques.

\section{Encadré 1 \\ Fumigation à la phosphine}

Il s'agit d'une technique basée sur l'usage d'un gaz biocide, praticable seulement par des personnes certifiées et employées par une société agréée. Le phosphure de magnésium ou d'aluminium solide, qui est utilisé, libère le gaz phosphine $\left(\mathrm{PH}_{3}\right)$ au contact de l'humidité de l'air. Ce gaz diffuse dans toute la masse, dans l'air interstitiel et également à l'intérieur des graines, et élimine tous les stades d'insectes (œufs, larves, adultes). Cela nécessite une bonne étanchéité de la structure de stockage pour pouvoir maintenir une concentration de gaz suffisante pendant une durée déterminée de quelques jours. Cette technique est donc réservée aux installations adaptées, qui doivent être rendues étanches. L'avantage de la fumigation à la phosphine est qu'elle ne laisse pas de résidus dans les graines en respectant le temps de désorption du gaz. Par contre, cette technique n'a qu'un effet curatif, le traitement n'ayant aucune rémanence après le dégazage. Elle permet une bonne éradication mais ne prévient pas contre une réinfestation. Cette technique est très peu utilisée actuellement en France car les structures de stockage ne sont pas adaptées et nécessiteraient pour cela des travaux d'étanchéification.

Une étude réalisée par le Cetiom a permis d'adapter cette technique au tournesol. Deux types de fumigation ont été testés : une fumigation de stockage qui permet d'éliminer tous les stades d'insectes avec une dose normale de $\mathrm{PH}_{3}\left(1,5 \mathrm{~g} / \mathrm{m}^{3}\right)$, et une fumigation d'agréage à dose faible $\left(0,045\right.$ à $\left.0,09 \mathrm{~g} / \mathrm{m}^{3}\right)$ dont l'action choc élimine seulement les insectes adultes pour des denrées rapidement vendues et transformées. Pour une fumigation de stockage, il faut attendre entre 3 et 30 jours de désorption pour que les résidus de phosphine dans les graines de tournesol soient inférieurs à $10 \mu \mathrm{g} / \mathrm{kg}$ selon les conditions de température et la durée de la fumigation. La fumigation d'agréage ne laisse pas de résidus au-delà de 24 heures, quelle que soit la température. Ces deux types de fumigation ont une bonne efficacité sur les espèces d'insectes rencontrées dans le tournesol. 


\section{Encadré 2}

Méthode rapide d'analyse de résidus de pesticides sur graines oléagineuses

De nombreuses méthodes existent pour la détermination des résidus de pesticides dans l'huile. Cependant, aucune étude ne s'est réellement penchée sur les résidus de pesticides présents dans les graines. Ces méthodes sont plus ou moins complexes, elles nécessitent une quantité considérable de solvant. L'inconvénient majeur était le passage obligatoire par l'huile pour pouvoir quantifier les pesticides dans les graines. Ces dernières années, le laboratoire du Cetiom à Ardon a axé ses recherches sur la mise au point d'une méthode d'analyse des résidus de pesticides directement dans les graines oléagineuses sans passer par l'huile. Les objectifs importants de cette méthode étaient qu'elle soit simple, rapide, économique et respectueuse de l'environnement. Une extraction solide-liquide avec de l'isooctane suivi d'une extraction liquide-liquide avec de l'acétonitrile permet d'extraire les résidus organophosphorés à partir des graines oléagineuses. Une purification sur cartouche C18 SPE a été optimisée. L'analyse des sept molécules organophosphorés est réalisée en chromatographie en phase gazeuse avec un détecteur thermoïonique. Les taux de récupération obtenus sont de l'ordre de 100 à $110 \%$.

Le même travail est mené dans ce laboratoire sur deux autres familles de produits phytopharmaceutiques : les organochlorés et les pyréthrinoïdes, qui feront l'objet d'une publication prochaine.

Cependant, dans le cas d'organismes stockeurs de grande capacité, les risques de transfert existent à d'autres niveaux également : bandes transporteuses traitées, passage de graines oléagineuses dans les circuits après des céréales traitées sur le circuit, traitement des boisseaux d'expédition, etc. donc on ne peut pas exclure ce risque pour les organismes stockeurs non plus. Une enquête est actuellement en cours évaluer ce phénomène, à l'aide d'une traçabilité précise de lots de tournesol et d'analyses d'échantillons constitués à l'expédition.

\section{Méthodes d'analyses des résidus de pesticides}

L'analyse des résidus de pesticides dans les graines oléagineuses n'est encadrée par aucune méthode normalisée, et elle est difficile du fait de la complexité de cette matrice. Aussi, dans le cadre de la mise en place du plan de surveillance de la qualité sanitaire des oléagineux, un groupe de travail animé par le Cetiom et I'Iterg a entrepris de développer une méthode d'analyse des résidus de pesticides dans les graines oléagineuses et les huiles en vue de sa normalisation (Afnor et CEN). Ce groupe réunit environ 25 laboratoires publics et privés et a sélectionné les méthodes les plus performantes à l'issue d'un essai circulaire organisé en 2004 où chaque laboratoire utilisait sa propre méthode. Les résidus étudiés, au chez une dizaine d'organismes stockeurs pour

nombre de 16 , sont des molécules organophosphorées, organochlorées et pyréthrinoïdes (dont les substances le plus souvent retrouvées dans les enquêtes). Les méthodes sélectionnées ont été compilées dans une méthode commune qui comporte plusieurs étapes :

- Extraction de la matière grasse des graines oléagineuses : à froid par un mélange de solvant, extraction à chaud type Soxhlet, ou par solvant à haute température sous pression.

- Pré-purification pour l'extraction des résidus de pesticides de la matière grasse : extraction par congélation, extraction liquide-liquide suivie d'une congélation ou d'une centrifugation cryogénique, ou chromatographie par perméation sur gel.

- Purification de l'extrait : sur cartouches commerciales de type SPE ou sur colonne de silice activée à $130^{\circ} \mathrm{C}$.

- Analyse par chromatographie en phase gazeuse couplée à un détecteur de masse ou des détecteurs spécifiques ou HPLC couplée à un détecteur de masse.

Cette méthode fait encore à ce jour l'objet d'essais circulaires afin que l'ensemble des laboratoires puisse la prendre en main et afin de vérifier qu'elle permet d'obtenir des résultats suffisamment fiables.

En parallèle, le Cetiom met au point une méthode rapide d'analyse des résidus de pesticides sur graines (encadré 2).

Tableau 2. Teneurs en résidus de pyrimiphos-méthyl (PMM) en $\mathrm{mg} / \mathrm{kg}$ dans les graines de colza et grains de blé stockés sur les plaques traitées pour deux durées de contact et taux de transfert* en \% (moyenne de 2 répétitions).

\begin{tabular}{|lccc|}
\hline & $\begin{array}{c}\text { Durée de } \\
\text { contact }\end{array}$ & $\begin{array}{c}\text { Teneur en résidus } \\
\text { de PMM }\end{array}$ & Taux de transfert \\
\hline Blé & 8 jours & 15 & $17,7 \%$ \\
(teneur initiale $0,062 \mathrm{mg} / \mathrm{kg}$ ) & 29 jours & 17,5 & $20,6 \%$ \\
Colza & 8 jours & 17,5 & $10,7 \%$ \\
(teneur initiale $0,028 \mathrm{mg} / \mathrm{kg}$ ) & 29 jours & 14,5 & $8,9 \%$ \\
\hline
\end{tabular}

*Taux de transfert $=[($ teneur finale dans les grains - teneur initiale dans les grains $) \times$ quantité de grains par plaque] / dose réelle de matière active par plaque utilisée lors du traitement.

\section{Conclusion}

L'ensemble de la filière oléagineuse a pris conscience des problèmes posés par les résidus $d^{\prime}$ insecticides et recherche des solutions de façon concertée. Le contexte réglementaire est en pleine évolution, ce qui devrait avoir une répercussion sur les résidus retrouvés dans les produits oléagineux. Le dichlorvos, insecticide de contact à effet choc, vient d'être interdit pour le traitement des céréales stockées. On peut imaginer que cela favorisera le développement de la fumigation au gaz phosphine, si les organismes stockeurs décident d'adapter leurs structures de stockage. L'utilisation du malathion et du pyrimiphos-méthyl, d'usage courant au stockage également, va peut-être être interdite. Seules les autorisations pour le chlorpyriphos-méthyl et la deltaméthrine ont été renouvelées à ce jour. Si l'utilisation de ces molécules se développe au stockage et en particulier celle de la deltaméthrine, cela risque de poser un problème pour la filière oléagineuse car des travaux menés par l'Iterg ont montré que cette molécule ne peut pas être complètement éliminée au raffinage contrairement aux insecticides organophosphorés (dichlorvos, malathion, pyrimiphos-méthyl, chlorpyriphosméthyl, fénitrothion) qui sont complètement éliminés lorsque les conditions de raffinage sont bien adaptées. On peut toujours envisager

Tableau 3. Extrapolation des résultats sur plaques à des cellules fermières pour le colza, pour un traitement des parois à $0,2 \mathrm{~g} / \mathrm{m}^{2}$ de pyrimiphos-méthyl.

\begin{tabular}{|c|c|c|c|}
\hline $\begin{array}{l}\text { Temps } \\
\text { de } \\
\text { contact } \\
\text { (jours) }\end{array}$ & $\begin{array}{c}\text { Type } \\
\text { cellule } \\
\text { (tonnes) }\end{array}$ & $\begin{array}{c}\text { Surface } \\
\text { cellule } \\
\left(\mathrm{m}^{2}\right)\end{array}$ & $\begin{array}{c}\text { Teneur } \\
\text { PMM } \\
\text { graines } \\
\text { calculé } \\
\text { (mg/kg) }\end{array}$ \\
\hline 8 & 10 & 25 & 0,059 \\
\hline 8 & 30 & 50 & 0,039 \\
\hline 29 & 10 & 25 & 0,049 \\
\hline 29 & 30 & 50 & 0,032 \\
\hline
\end{tabular}


qu'il y ait une homologation d'un insecticide pour traiter les graines oléagineuses stockées, mais aucune démarche n'est entreprise en ce sens à ce jour. Sur la base des enquêtes faites en organismes stockeurs, qui cherchent à évaluer l'importance des contaminations fortuites par contact avec des parois et matériaux traités ou ayant véhiculé des céréales traitées, on peut également envisager de faire évoluer les limites réglementaires afin de prendre en compte ce phénomène, sans pour autant mettre ces limites à des niveaux qui permettraient des traitements des graines. Ces enquêtes faites sur le terrain, en collaboration avec l'ensemble des organisations de la filière, sont un bon outil de communication entre opérateurs et donnent une base d'argumentation avec les professionnels, qui permettra de faire évoluer les pratiques [1-8].
Ces travaux ont reçu le soutien financier de I'ONIGC dans le cadre du Plan de relance des oléagineux.

\section{RÉFÉRENCES}

1. DARRACQ $S$. La sécurité sanitaire : une priorité pour la filière oléagineuse. Oléoscope 2004 ; 74 : 34-6.

2. DAUGUET S, LACOSTE F. La qualité sanitaire des graines, tourteaux et huiles. Oléoscope $2005 ; 82: 20-2$.

3. DAUGUET S, LOISON JP, FLEURAT-LESSARD F. Insectes de stockage des graines de tournesol et de colza : détermination des espèces présentes en France et facteurs de risque. Phytoma $2005 ; 586: 24-8$.

4. DELPLANCKED. Sécurité sanitaire des aliments: de la nécessité d'une approche de filière. OCL $2000 ; 7(5)$ : 411.
5. LACOSTE F, et al. Contrôle des composés indésirables dans les huiles végétales et mise en place d'observatoires. OCL $2005 ; 12(5-6)$ : 372-7.

6. LACOSTE F, DAUGUET S, SOULET B, ARNAUD JN, BRENNE E. Food safety in the field of vegetable oils: from monitoring of undesirable compounds to survey plans. 26th ISF World Congress, Prague, 25-28 September 2005.

7. LOISON JP, DAUGUET S. Comment limiter le risque d'insectes au stockage. Oléoscope 2005 ; $82: 4-5$.

8. PAGES X, BIROT C, ESCABASSE J, et al. 2006. Refining: removal of pesticides residues. 4th Euro Fed lipid Congress, Madrid, 1-4 October 2006. 